\title{
Perfil de la demanda del turismo receptivo durante la temporada de Semana Santa en el Estado Mérida, Venezuela*
}

\author{
MARYSELA COROMOTO MORILLO MORENO** \\ CARLOS GUILLERMO QUINTERO GULL***
}

* Artículo resultado del proyecto de investigación financiado por el CDCHTA de la Universidad de Los Andes (№ E-377-16-09-A).

** Doctora en Ciencias Económicas y Empresariales. Universidad de Los Andes, Mérida, Venezuela. E-mail: morillom@ula.ve. ORCID: 0000-0003-0697-4677. Google Scholar: https://scholar.google.com/citations?user=DsJhEwYAAAAJ\&hl=es.

*** Magíster en Modelado y Simulación de Sistemas. Universidad de Los Andes, Mérida, Venezuela. E-mail: cgull@ula.ve. ORCID: 0000-0001-9575-0020. Google Scholar: https://scholar.google.co.ve/citations?hl=es\&user=n4-_6jIAAAAJ. 


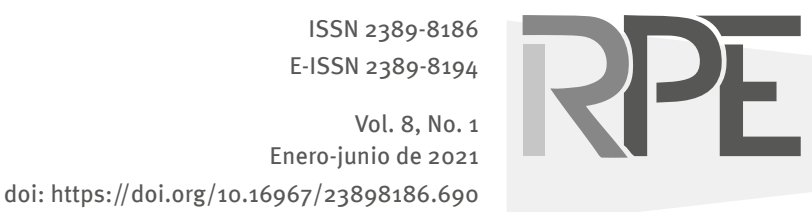

COMO CITAR ESTE ARTÍCULO

How to cite this article:

Morillo, M.y Quintero, C. (2021). Perfil de la demanda del turismo receptivo durante la temporada de Semana Santa en el Estado Mérida, Venezuela. Revista Perspectiva Empresarial, 8(1), 101-112.

Recibido: 11 de febrero de 2021 Aceptado: 30 de junio de 2021
RESUMEN Objetivo. Analizar el perfil del turismo receptivo merideño durante la Semana Santa de los últimos años; temporada que concentra la mayor cantidad de manifestaciones y acontecimientos culturales y religiosos del Estado. Metodología. A partir de un análisis multivariante de correspondencia múltiple se hallaron cuatro perfiles sobre la base de variables diferenciadas significativamente. Resultados. En Venezuela, incluido el Estado Mérida, urge impulsar múltiples tipologías de turismos especializados basados en su patrimonio histórico-cultural y religioso; por ello se recomienda el fortalecimiento y la combinación de los atractivos turísticos. Conclusiones. Se concluye que la demanda turística en el Estado Mérida durante la temporada objeto de estudio es moderadamente homogénea, en donde una considerable cantidad de turistas mostró motivos de visita diferentes al aspecto religioso.

PALABRAS CLAVE turismo religioso, competitividad, patrimonio histórico y cultural.

\section{Profile of inbound tourism demand during the Easter Week season in the State of Mérida, Venezuela}

ABSTRACT Objective. To analyze the profile of Merida's inbound tourism during Easter Week in recent years; a season that concentrates the largest number of cultural and religious events and manifestations in the State. Methodology. Based on a multivariate analysis of multiple correspondence, four profiles were found on the basis of significantly differentiated variables. Results. In Venezuela, including the State of Merida, there is an urgent need to promote multiple typologies of specialized tourism based on its historical-cultural and religious heritage; therefore, the strengthening and combination of tourist attractions are recommended. Conclusions. It is concluded that tourism demand in the State of Mérida during the season under study is moderately homogeneous, where a considerable number of tourists showed reasons for visiting other than the religious aspect.

KEYWORDS Religious tourism, competitiveness, historical and cultural heritage. 


\section{Perfil da demanda por turismo receptivo durante a temporada da Semana Santa no Estado de Mérida, Venezuela}

RESUMO Objetivo. Analisar o perfil do turismo receptivo em Mérida durante a Semana Santa nos últimos anos; temporada que concentra o maior número de manifestações e eventos culturais e religiosos do Estado. Metodologia. A partir de uma análise de correspondência múltipla multivariada, quatro perfis foram encontrados com base em variáveis significativamente diferenciadas. Resultados. Na Venezuela, incluindo o Estado de Mérida, é urgente promover os múltiplos tipos de turismo especializado a partir de seu patrimônio histórico-cultural e religioso; por este motivo, recomendase o fortalecimento e combinação de atrativos turísticos. Conclusões. Conclui-se que a demanda turística no Estado de Mérida durante a temporada em estudo é moderadamente homogênea, onde um número considerável de turistas apresentou motivos de visita diferentes ao aspecto religioso.

PALAVRAS CHAVE turismo religioso, competitividade, patrimônio histórico e cultural. 


\section{Introducción}

Al reflexionar sobre la actividad turística es ineludible partir de la relevancia de la misma como elemento clave para el progreso socioeconómico gracias a su estrecha vinculación con el desarrollo de los destinos. Ello se refleja en el número de personas movilizadas por diversos motivos y en los elevados niveles de gasto turístico con un indetenible aumento (OMT, 2020); esto hace que la actividad sea una alternativa, incluso para las regiones menos desarrolladas. Según la OMT (2018) - pese a las fuertes conmociones mundiales de diversa índole (económica, sociales, políticas, ambientales entre otras) - el turismo representa el $10 \%$ del PIB mundial, el $30 \%$ de los servicios de exportación y el $7 \%$ del total de exportaciones de bienes y servicios. A 2019, las llegadas de turistas internacionales en todo el mundo crecieron un $4 \%$ para alcanzar 1500 millones de turistas; tasa relativamente alta; al igual que los años anteriores, 2017 y 2018 con un $+7 \%$ y $+6 \%$ respectivamente (OMT, 2020).

En los últimos años - a la par de los adelantos de los medios de comunicación y de transportela evolución del sector turístico ha dado respuesta a los cambios de sus consumidores, a sus estilos de vida, a su nivel educativo, a su tiempo y renta disponible; atiende a turistas experimentados, altamente informados y educados, no solo sobre los lugares de interés y alternativas de viaje (medios de transporte, forma de pago, alternativas de alojamiento) sino en sostenibilidad y conservación de recursos; producto de su insaciable deseo de aprender, nutrir y actualizar sus conocimientos y experiencias mediante el intercambio cultural. Hoy los turistas poseen diversidad de necesidades y deseos, mayor sensibilidad hacia la preservación medioambiental y cultural y motivaciones más complejas que buscan crecimiento personal a partir de experiencias enriquecedoras al considerar y valorar no solo el entorno natural sino la cultura del destino turístico. Tal diversidad se refleja en sus múltiples motivos de viaje. Esta tendencia ha sido recurrente desde los años 90, según Crouch (1994); tendencia que continua vigente en estudios recientes realizados por Wang, Xiang y Yunpeng (2013) y Hsin-Yu (2021).
Dichas tendencias de motivo de viaje se han repetido alrededor del mundo en últimas décadas (OMT, 2018), por lo que el planificador y empresario turístico deberá adaptarse al nuevo turista y a la nueva concepción del producto ofrecido no solo a partir de su poder de negociación sino de la variedad de combinaciones del producto turístico, incluyendo sus elementos básicos (alojamiento, restauración, transporte entre otros). Por ello hoy es necesaria la diversificación de los atractivos turísticos a la par de una adecuada segmentación del mercado a partir no solo de variables demográficas, profesiones y estilos de vida sino de intereses concretos (Garau-Vadell, GutierrezTaño and Diaz-Armas, 2018; Mariani et al., 2014).

En Venezuela, incluido el Estado Mérida, datos recientes muestran la complejidad de la situación socioeconómica que apuntan a su diversificación en torno a tipologías de turismos especializados según las tendencias mundiales y las potencialidades de cada región como alternativas de oferta.

A nivel local, el Estado Mérida — ubicado en la región andina venezolana- pese a que el turismo es considerado como actividad económica estratégica dentro de planes de desarrollo regionales y nacionales, tal énfasis no ha logrado su cometido; pues en los últimos años (2000-2018) las cifras mostradas por la Corporación Merideña de Turismo - CORMETUR - la actividad ha mostrado las mismas tendencias y debilidades como el origen nacional de la mayoría de turistas, su breve estadía y traslado terrestre; todo esto impulsado solo por el recreo, las bellezas naturales, el clima y los precios bajos, lo cual ha contribuido a su escaso desarrollo. En estos casos, García y Olmos (2015) recomiendan la diversificación de la oferta turística como estrategia para revitalizar destinos maduros o de larga tradición. Es por ello que en el Estado Mérida con vista a incrementar la duración promedio de la estadía del visitante es necesario desarrollar atractivos turísticos, motivadores o impulsadores de visitas capaces de atraer y prolongar la estadía de un mayor número de turistas, incluyendo las temporadas bajas, lo cual podría elevar el gasto turístico durante todo el año.

El Estado Mérida es una de las entidades de mayor riqueza cultural y religiosa en sus espacios públicos, como parte de su identidad histórica y cultural, a ser usada en la diversificación turística 
(Boada, 2018); al igual que infinidad de destinos turísticos en el mundo que han logrado posicionarse y diferenciarse con una adecuada planificación y gestión de sus atractivos culturales y religiosos. Por ello apoyados en los múltiples atractivos del patrimonio histórico-cultural del Estado Mérida, en algunas tendencias y caracterizaciones de las últimas temporadas asociadas al turismo religioso (época de realización de abundantes celebraciones religiosas y culturales) y con vista a la urgente necesidad de potenciar y diversificar los atractivos turísticos merideños (configurados desde hace décadas como un destino turístico recreacional paisajístico de montaña) se considera pertinente enfatizar las bondades y fortalezas del Estado para la práctica del turismo cultural y religioso (Argyriadis, 2008).

Como requisito previo alasfases de planificación y promoción para el adecuado desarrollo del turismo cultural y religioso merideño, así como alternativa de diversificación de la oferta turística de la entidad, el presente trabajo intentó analizar el perfil del turismo receptivo merideño durante la Semana Santa o Semana Mayor de los últimos años; temporada que concentra la mayor cantidad de manifestaciones y acontecimientos culturales y religiosos del Estado.

A continuación se abordan los principales fundamentos teóricos sobre el turismo cultural y religioso, seguido del diseño metodológico requerido para la obtención de los resultados y las conclusiones presentadas en el estudio.

\section{Marco teórico}

En la planificación turística la oferta está representada por la totalidad de servicios y de bienes a ser consumidos por los turistas y residentes, comercializados en un determinado espacio geográfico y sociocultural, con un valor o atractivo a disposición de un mercado competitivo. Esa oferta está compuesta por la planta, la infraestructura turística y los atractivos. Estos últimos son "la expectativa de ver o hacer algo concreto [...] que mueven a turistas y excursionistas a desplazarse a un destino turístico" (Sancho, 2006, p. 134); estos son la verdadera razón por la que los viajeros se desplazan a un determinado destino; además son los responsables de la elección entre un lugar u otro como elemento activador del desplazamiento y pueden estar constituidos por el sitio o evento que se desea conocer, visitar o presenciar; de ahí que los atractivos puedan ser de tipo natural y cultural.

Los atractivos culturales están asentados en centros urbanos o rurales, según el patrimonio cultural de la localidad. El patrimonio cultural es "el conjunto de bienes, materiales e inmateriales, [...] portadores de valores culturales propios de la comunidad, [...] (de) [...] alto contenido simbólico, [...] merecedores de una especial protección [...] (o) conservación [...] (y) [...] uso" (Velasco, 2009 , p. 238). Este conjunto de bienes incluye las manifestaciones históricas, artísticas y hasta ambientales; estas constituyen la esencia de los lugares (reflejada en sus paisajes, sus historias, leyendas, cuentos y costumbres) (Boada, 2018); también incluye elementos que caracterizan a los lugareños en su manera de hacer, de ser, y de relacionarse desde su cotidianeidad e incluso a través de sus imaginarios. Esta versatilidad de manifestaciones - que terminan por definir la identidad de todo pueblo- puede ser percibida y disfrutada a través de los sentidos en sonidos naturales, creados o recreados, en la cocina, en lo que se observa o se toca al ser creados (construcciones, artesanías, obras entre otras).

Para el turismo el patrimonio (ya sea cultural, natural o paisajístico) es la materia prima para una adecuada planificación que garantice su uso y disfrute sostenible para la comunidad receptora y visitantes (conservación, estudio y valorización) (Boada, 2018). Los atractivos turísticos pueden ubicarse en los lugares arqueológicos (petroglifos, cementerios, huacas y mintoyes, lugares de observación, paradores, miradores y yacimientos), en los monumentos con manifestaciones culturales e históricas (iglesias, capillas, conventos, fuertes, fortalezas, plazas, puentes, represas, embalses, sitios históricos, industrias entre otros), en las construcciones (religiosas, militares, gubernamentales, civiles y conmemorativas) y en los centros culturales y artísticos (teatros, museos, bibliotecas, casas de historia y de cultura) (Morillo, 2014; Maxim and Chasovschi, 2021), dispuestos a modo de instituciones públicas o privadas que promueven y difunden la cultura (Hughes, 1995). Otro importante atractivo cultural de la oferta 
turística es el folclore (Goeldner y Ritchie, 2011), el cual consiste en el conjunto de intangibles (creencias, leyendas y costumbres tradicionales de la localidad receptora); este se expresa en la cultura popular, en las manifestaciones artesanales, las fiestas tradicionales y festividades populares, en las creencias mágico-religiosas, las danzas, bailes populares, juegos, literatura oral tradicional, las leyendas y cuentos y la gastronomía tradicional y/o popular (Morillo, 2014).

Al combinar el abanico de atractivos culturales, religiosos e históricos se origina una tipología de turismo llamado turismo cultural y religioso (Swarbrooke, 1995; Ya-Ping Chang and KuangPeng, 2021); este se basa en atracciones creadas capaces de movilizar a visitantes como son los festivales folclóricos, artísticos, mercados y ferias, aniversarios y eventos religiosos principalmente. Para McIntosh y Goeldner (1986) y MostafaRasoolimanesh etal. (2011) se viaja para observar expresiones culturales, estilos de vida, costumbres, danzas, rituales religiosos y ceremonias. Esto puede incluir el turismo étnico y las costumbres típicas, rituales y estilos de vida de indígenas en espacios o contextos primitivos, vírgenes o puros al menos para el turista y por lo tanto genuinos a su vista, generando un encuentro de culturas distintas.

Así pues, el turismo religioso se "vincula a las prácticas religiosas en lugares determinados con un significado religioso" (Aulet y Hakobyan, 2011, p. 64). Mientras que Carrizo y Vieira (2009) y Morillo (2018) lo conciben como el desplazamiento de personas interesadas en presenciar actos, acontecimientos, eventos o celebraciones religiosas; al igual que conocer lugares, monumentos, templos o personajes que simbolizan un credo con el cual el turista se identifica; es un turismo de afinidad, ejemplificado a gran escala por las movilizaciones realizadas por fieles a lugares sagrados para el cumplimiento de sus compromisos de credo en fechas especiales (romerías y peregrinaciones). Shinde (2010) lo asocia a los lugares de peregrinación, entendida esta última como un viaje a lugares santos por autoconocimiento o espiritualidad (Collins, 2010). Es un turismo que inicia desde tiempos bíblicos con las peregrinaciones a sitios sagrados (Tobón y Tobón, 2013), de gran crecimiento mundial por su impacto en la economía local y global dado que las movilizaciones contabilizan no solo al peregrino (fiel que ejerce penitencia, devoción y oración), sino también al viajero de comportamiento o interés distinto (cultural, por conocimiento, recreación, ocio, descanso o curiosidad); de ahí que tan solo los centros de culto cristiano más grandes del mundo movilizan casi 25 millones de personas, incluyendo fieles de otras religiones (Aulet y Hakobyan, 2011). El término de turismo religioso es reciente y de elevada complejidad al conjugar y combinar características y elementos de conceptos distintos (turismo y religión), motivos del viaje (penitencia, devoción o por conocimiento cultural) y recorridos del itinerario, desviado muchas veces al turismo de ocio u otros. Por tal motivo el turismo religioso desde los años 50 y 60 ha sido reflexionado y estudiado desde lo artístico, etnográfico, eclesiástico, económico, antropológico, sociológico y geográfico (Aulety Hakobyan, 2011).

El turismo religioso está estrechamente vinculado al turismo cultural y de eventos. El primero se desarrolla alrededor del patrimonio cultural e histórico de los destinos (Morére y Perelló, 2013), lo que incluye festivales folclóricos y religiosos. Es así que los principales destinos religiosos son lugares santos configurados como sitios de devoción y peregrinaje y como asentamientos de monumentos arquitectónicos de gran valor histórico-cultural. Desde esta óptica "el turismo religioso se compone de patrones religiosos, culturales, tradicionales, espirituales, paisajísticos, que interactúan en la intención y la decisión de viaje" (Aulet y Hakobyan, 2011, p. 63). La vinculación con el turismo de eventos (reuniones o concentraciones de grupos afines) se explica porque las festividades religiosas incluyen la concentración de viajeros o grupos afines identificados o interesados en creencias o eventos de credos (Pérez, 2014); es decir que se movilizan por devoción, penitencia o para enriquecer sus valores históricos y culturales (Tobón y Tobón, 2013). De esta forma los destinos de peregrinación se han convertido recientemente en lugares que atraen a peregrinos y turistas en general con motivaciones y expectativas radicalmente distintas dada la multifuncionalidad de sus espacios (Aulet y Hakobyan, 2011).

Tales intereses, con constantes movilizaciones, han generado infinidad de exitosos destinos turísticos (Ciudad del Vaticano, la catedral de San Patricio en Nueva York, La Meca, los monasterios 
budistas en China, Tierra Santa en Jerusalén, Santiago de Compostela entre otros) (CollinsKreiner, 2010); con ubicaciones principales en Italia, Israel, Grecia, Portugal, Finlandia, México, Francia, Brasil y España (Nieminem, 2012); diferenciados por sus atractivos culturales y religiosos, lo que se constituye en motivo de viaje para una gran cantidad de personas (Collins-Kreiner and Kliot, 2000). Aunque el turismo religioso ha sido erradamente dirigido hacia el segmento de viajeros dela tercera edad y de bajos ingresos dada su escasa diversión, austeridad de servicios (alimentación y alojamiento) y breve permanencia, lo que amerita una adecuada gestión.

\section{Metodología}

Para el alcance del objetivo trazado se acudieron a diversas fuentes para conceptualizar, describir, descomponer y explicar el tema del turismo religioso y cultural, incluyendo documentos emitidos por la OMT y CORMETUR para describir y analizar la actividad turística a nivel local e internacional. Con ello se logró conformar las bases conceptuales y los antecedentes expuestos que permitieron a la vez analizar los resultados.

Seguidamente se diseñó una investigación no experimental con base en las encuestas de flujo turístico de perfil del turista y de salida desarrollada y aplicada por la Unidad Estadística de la Gerencia de Promoción y Mercadeo de la CORMETUR con el objetivo de recopilar información sobre la actividad turística merideña, su situación, la cantidad de turistas recibidos y sus necesidades de esparcimiento, para el diseño de estrategias de marketing que contribuyan al fortalecimiento del sistema turístico merideño. Específicamente la primera encuesta estimó la cantidad de turistas que ingresan al Estado Mérida en la temporada, según su localidad o país de residencia y el tamaño promedio del grupo. Las encuestas de perfil y de salida midieron variables sociodemográficas (edad, género, estado civil, nivel educativo, ocupación actual, forma de viaje (en grupo o en tours), motivo de viaje, atracciones especiales de visita, medios de información e influenciadores, medios de transporte, duración promedio y lugar de estadía, pueblos y lugares visitados, actividades realizadas, tipo de alojamiento entre otros) y la opinión del turista sobre el nivel de precios y calidad de los servicios.

La población muestreada estuvo constituida por los turistas recibidos en el Estado Mérida durante la Semana Santa de 2014, 2015, 2016 y 2017; temporada alta de 9 días durante los meses de marzo y abril, en observancia de las fiestas móviles del calendario litúrgico católico mundial; sobre la cual se muestrearon las variables del jefe del grupo (edad, sexo, nivel educativo, ocupación, estado civil y opinión sobre los servicios) y variables grupales (tamaño de grupo, tipo de transporte y alojamiento y lugar de procedencia).

A partir de un muestreo estratificado probabilístico con un margen de error del $5 \%$, los estratos correspondieron a los distintos sitios donde se aplicaron las encuestas como puntos de control turístico del Estado tales como alcabalas terrestres, terminales aéreos y terrestres y puntos de interés o de alta afluencia turística. Los puntos de control o sitios de aplicación de las encuestas dependieron de los objetivos de cada una; de esta manera las encuestas de flujo turístico y de perfil salida tuvieron como puntos de recolección las cuatro alcabalas limítrofes del Estado Mérida (La Mitisus, Timotes, El Quebradón y el Peaje Zea), el Aeropuerto Internacional Juan Pablo Pérez Alfonzo de la cuidad de El Vigía y en los terminales terrestres de pasajeros José Antonio Paredes en la ciudad de Mérida y el de El Vigía; mientras que la encuesta de perfil del turista se aplicó en siete sitios de interés turístico (Parque Zoológico Chorros de Milla, Mercado Principal, Parque Temático La Venezuela de Antier, Museo de Ciencia y Tecnología, Plaza Las Heroínas, Sistema Teleférico Mukumbarí, Capilla de Piedra de Juan Félix Sánchez y Parque Beethoven).

Posteriormente se describieron las características y aspectos clave del comportamiento de la afluencia turística durante las temporadas mencionadas como variables manifiestas $\mathrm{u}$ observables y se realizó un análisis multivariante de correspondencia múltiple, utilizando el software $\mathrm{R}$ a través del uso particular del comando PRINCOMP. 


\section{Resultados y discusión}

De acuerdo a Guillen y Pérez (2016) y Boada (2018) el Estado Mérida posee grandes riquezas culturales debido a su larga historia y grandes acontecimientos que han marcado la sociedad y múltiples espacios públicos, lo cual ha pasado a construir la identidad de la localidad y a convertirse en su patrimonio histórico-cultural, lo que al día de hoy se considera como atractivos geoturísticos; esto es evidenciado en el Inventario de Patrimonio Cultural e Histórico del Estado Mérida efectuado por CORMETUR en 2013. Todos los monumentos y manifestaciones mencionadas, religiosas en su mayoría, atraen a gran cantidad de turistas; constituyendo a Mérida como un importante centro de interés turístico en determinadas épocas del año.

El análisis descriptivo de los datos de afluencia turística para el período 2000-2017 destaca que durante la Semana Santa arriban al Estado la segunda cantidad de turistas más elevada (29\% en promedio de la totalidad de turistas anuales), solo superada durante la temporada de vacaciones escolares con un $32 \%$; pese a su breve duración (9 días) frente a otras temporadas tales como Navidad y vacaciones escolares, 30 y 60 días respectivamente (figura 1 ).

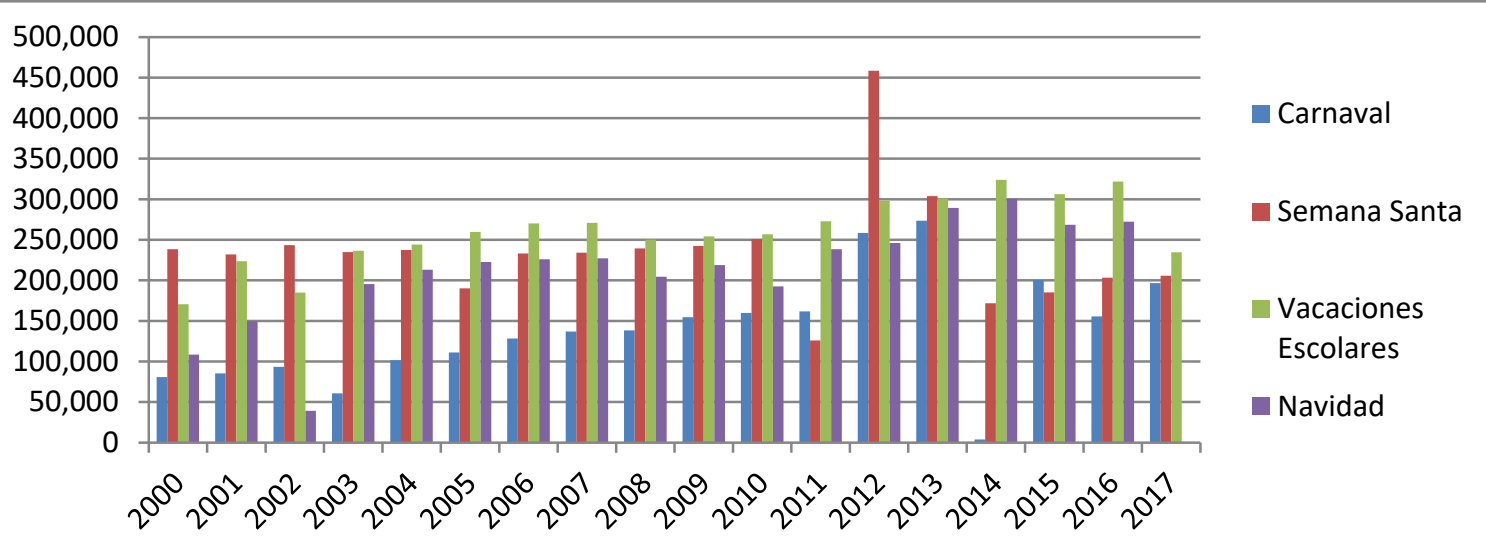

Figura 1. Afluencia de turistas al Estado Mérida por temporadas entre 2000 y 2017. Fuente: elaboración propia por parte de los autores.

El principal motivo de visita durante la temporada estudiada fue el recreo y las vacaciones con un promedio porcentual del $74,78 \%$, seguido de la visita a familiares y amigos con un $14,58 \%$; en contraste con la escasa proporción de viajeros por motivos religiosos, pese a que los encuestados podían seleccionar varias alternativas de respuesta (figura 2). Esta escases es paradójica, siendo explicada por Aulet y Hakobyan (2011) al indicar que dentro del turismo religioso se contabilizan o movilizan personas con otros motivos (festividad, lo cultural y natural, curiosidad, ocio y descanso). 


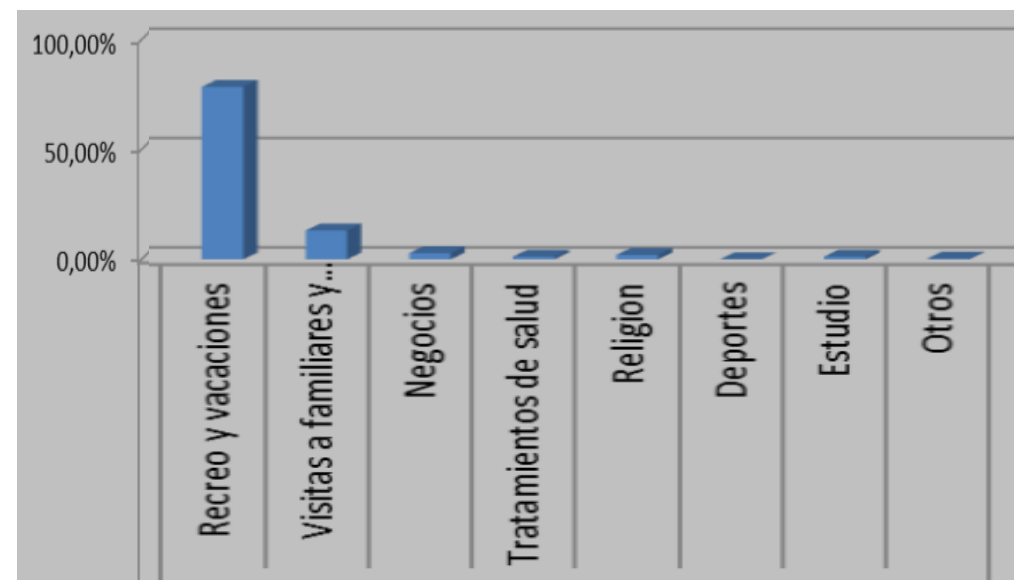

Figura 2. Motivos de visita al Estado Mérida en la temporada de Samana Santa entre 2000 y 2017. Fuente: elaboración propia por parte de los autores.

En lo que se refiere al tamaño de grupo de turistas, cabe destacar que la mayor proporción viaja en grupos de 4 y 6 personas; el $62 \%$ de los grupos esta entre 2 y 6 personas, obteniendo un valor promedio de 7,5 personas por grupo. A pesar de que la mayoría de los grupos se conformaron entre 2 y 6 personas tambíen se hallaron grupos con más de 20 personas, por lo que el promedio se inclina a valores extremos. Sobre el nivel educativo del jefe del grupo familiar se observó que más del $60 \%$ de los entrevistados tienen un nivel educativo universitario (incompleto y completo) seguido de secundaria completa (19\%) (figura 3).

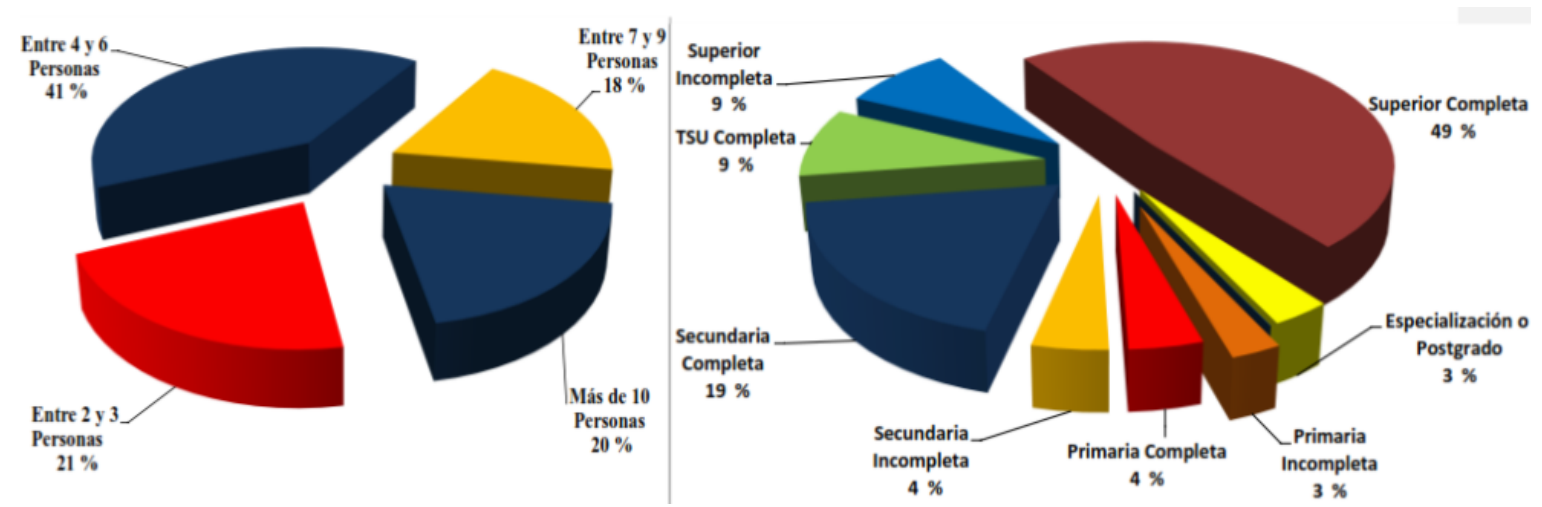

Figura 3. Tamaño de grupo y nivel educativo del jefe de grupo en la temporada de Samana Santa entre 2014 y 2017. Fuente: elaboración propia por parte de los autores.

El análisis descritivo sobre las características grupales (lugar de procedencia, tipo de alojamiento y medio de transporte) evidenció un patrón similar en cuanto al lugar de procedencia hacia las regiones centrales del país (Distrito Capital, Estados Aragua y Carabobo) (34\%), Occidental (Estados Lara, Zulia,
Táchira) (30\%) (figura 4). El medio de transporte más utilizado fue el carro particular y el autobus con más del $80 \%$; además se halló similitud en la alternativa de alojamiento entre la posada, el hotel y la casa de familia al representar en promedio el $80 \%$ (figura 5). 


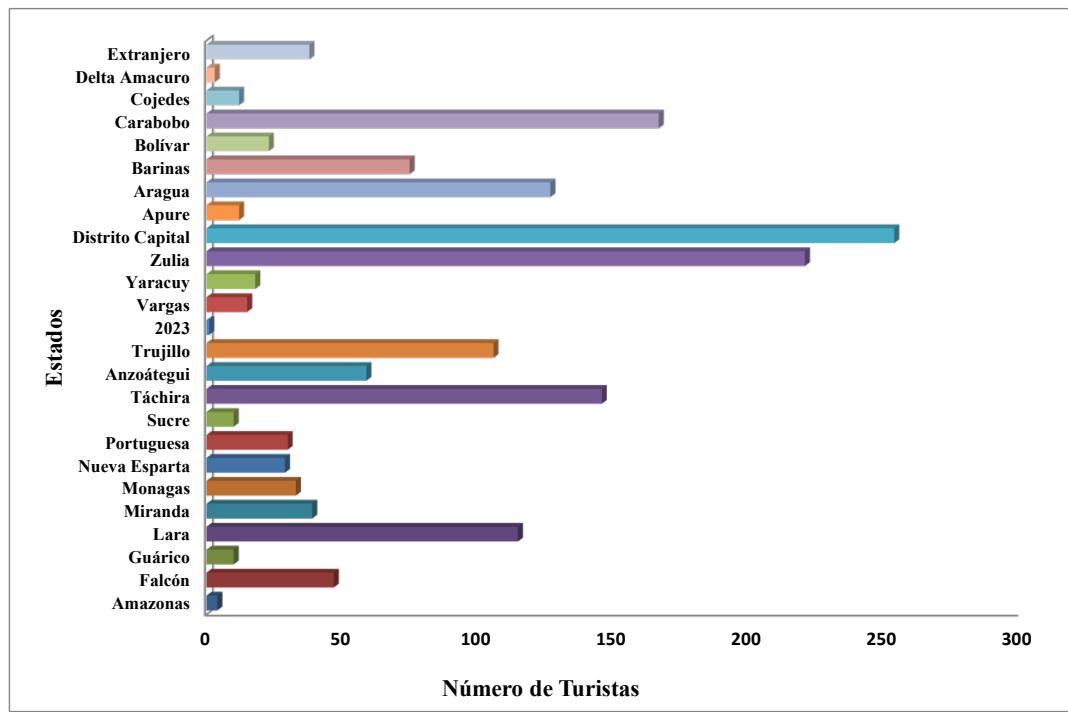

Figura 4. Entidad de procedencia de turistas al Estado Mérida en la temporada de Samana Santa entre 2000 y 2017. Fuente: elaboración propia por parte de los autores.
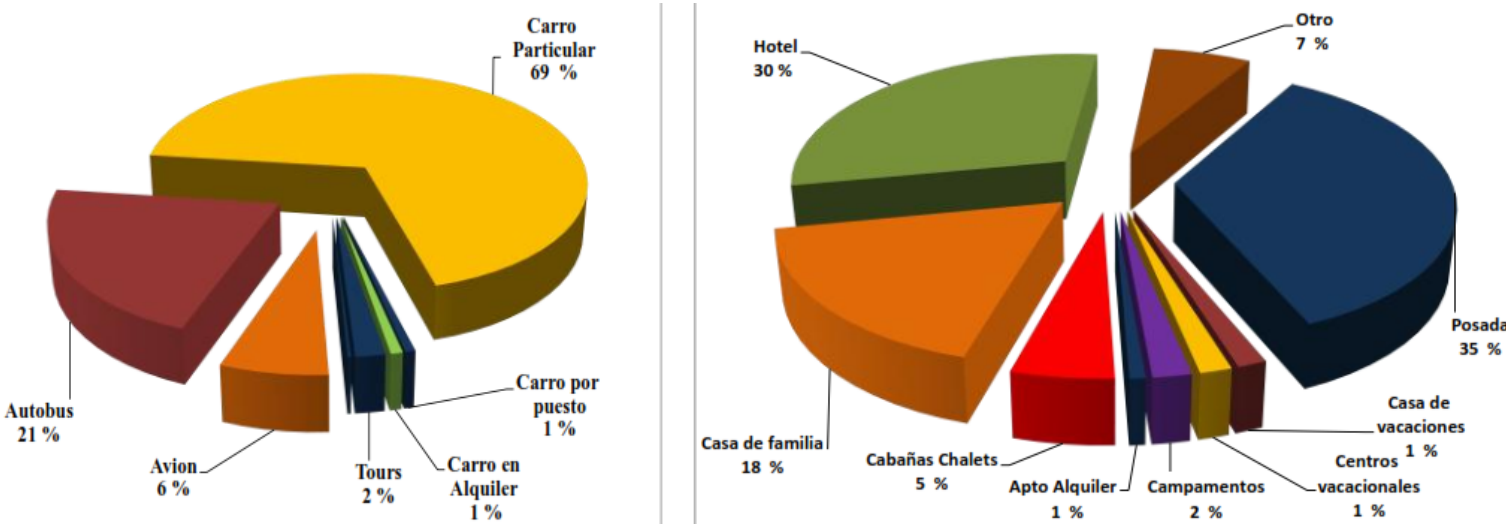

Figura 5. Medio de transporte y alojamiento utilizado por los turistas del Estado Mérida en la temporada de Samana Santa entre 2000 y 2017 . Fuente: elaboración propia por parte de los autores.

Con la finalidad de realizar una caracterización interanual de los turistas que visitaron el Estado Mérida en la temporada de Semana Santa entre 2014 y 2017 se practicó un análisis multivariante de correspondencia múltiple desde la óptica de la demanda turística. En dicho análisis, se halló una variabilidad total explicada del $60,82 \%$ en la que el Estado Mérida es receptor de un importante grupo de turistas nacionales que de acuerdo a sus características se pueden agrupar en cuatro perfiles. Un primer grupo de turistas (A) proveniente de los Estados Aragua, Lara y Carabobo de nivel educativo elevado (universitario completo), que viajan en grupos más grandes o medianos (entre 5 y 10 personas) trasladados en carro particular y alojados en hoteles y posadas; otra característica significativa es su estado civil, soltero o viudo. Un segundo grupo de turistas (B), totalmente distinto, que proviene del extranjero y alojados en carpas y en casas de vacaciones que utilizan la motocicleta como medio de transporte y cuyo estado civil es divorciado (figura 6). Este grupo de turistas, al no estar asociado de manera marcada a ningún año, hace posible afirmar que estuvo asociado en todos los años analizados. 
Otro grupo de turistas se halló en 2015 (C), el cual contrasta también con los grupos anteriores. Específicamente se ubicó un grupo de turistas en su mayoría provenientes del Occidente del país (Estados Táchira, Zulia y Trujillo) con un nivel educativo de bajo a moderado (primaria o secundaria), en grupos grandes (mayores a 10 personas), alojados en casas de alquiler o centros vacacionales y traslados predominantemente en carro por puesto o tours. Un cuarto grupo (D) se evidenció durante 2016 como viajeros en grupos pequeños y moderados (entre 2 y 5 personas), de nivel educativo mediano (técnico superior universitario o secundaria completa), que provienen de diversos Estados del país (incluido el Distrito Capital), de estado civil casado, alojados en apartamentos de alquiler, resort o casas de familia y trasladados en bus (figura 6).

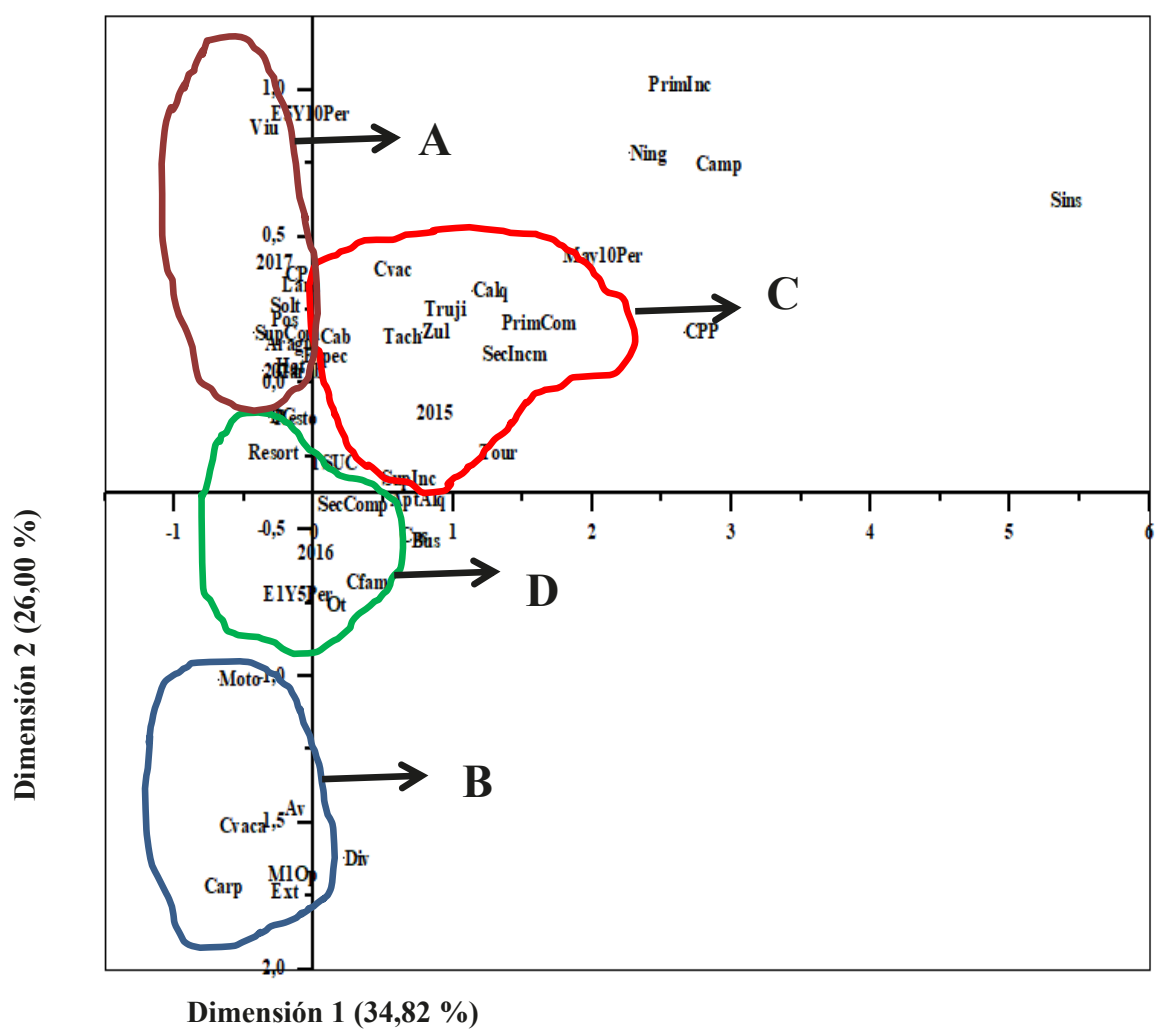

Nota: variabilidad total explicada del 60,82 \%.

Figura 6. Caracterización de los turistas en el Estado Mérida en la temporada de Samana Santa entre 2000 y 2017. Fuente: elaboración propia por parte de los autores.

\section{Conclusiones}

De cara a la diversificación de los atractivos turísticos y la revitalización del Estado Mérida como destino turístico religioso se analizó el perfil del turismo receptivo merideño durante la temporada de Semana Santa entre 2014 y 2017, temporada que concentra la mayor cantidad de manifestaciones y acontecimientos culturales y religiosos del Estado. Se halló durante la temporada de Semana Santa, de apenas 9 días de duración, que el Estado Mérida recibe la mayor cantidad de turistas solo superada por la cantidad de arribos experimentada en la temporada de vacaciones escolares (de 60 días de duración) y por encima de la cantidad de turistas que se recibe en la temporada de Navidad (de 30 días de duración). Destaca que 
el principal motivo de viaje no es precisamente el religioso (devoción y oración) sino la recreación y la visita a familiares y amigos, lo que muestra que las cifras del turismo religioso incluyen variedad de motivos de visita (Aulet y Hakobyan, 2011). Este comportamiento debe ser utilizado para robustecer el turismo receptivo merideño, en el sentido de potenciar y combinar los atractivos religiosos y los asociados al descanso y la recreación para atraer a una mayor cantidad de visitantes y prolongar su estadía al ofrecer mayores alternativas de comodidad o disfrute tanto para los peregrinos como al turista en general; todo alrededor de las festividades religiosas y nunca como un intercambio de atractivos, producto de un criterio o sentido de sostenibilidad para la preservación del patrimonio cultural e histórico del destino.

Además se evidenció el elevado nivel educativo de los jefes del grupo. Esto apunta a promover, usar y preservar la riqueza del patrimonio cultural e histórico del Estado; la cual es más valorada por los turistas de mayor nivel educativo y capaces de manejar mayor cantidad de información.

A partir de la caracterización interanual de los turistas que visitaron el Estado Mérida se lograron ubicar cuatro grupo de turistas distintos. Esos grupos, con un enfoque estratégico de diversificación de productos, pueden ser observados como interesantes y atractivos segmentos de mercado turístico; lo anterior, como una tipificación de la demanda del mercado en subgrupos o clases cada una debidamente caracterizada.

Aun cuando cada uno de los cuatro perfiles hallados poseen características distintivas se puede concluir que la demanda turística en el Estado Mérida es moderadamente homogénea; esto debido a la elevada cantidad de variables analizadas (sociodemográficas y jefe del grupo) sobre las cuales no se hallan diferencias significativas en cada grupo, siendo pocas las variables que permiten diferenciar los perfiles o segmentar la demanda turística. Durante las temporadas analizadas solo se pudieron hallar perfiles o grupos de turistas distintos principalmente a partir del tamaño de grupo, medio de transporte, alternativa de alojamiento, procedencia y nivel educativo del jefe del grupo; estas son las únicas variables que permiten definir tipologías de turistas.
En la mayor parte deperfiles (A, Cy D) se hallaron caraterísticas comunes, lo que revela la configuración de un perfil común de turistas presentes en todo el período analizado. A partir de la considerable cantidad de turistas, que viajan por motivos no precisamente religiosos y con nivel educativo alto, se apunta a la diversificación y combinación de atractivos y a la revitalización del Estado Mérida como destino turístico de naturaleza-montaña a través del desarrollo de rutas geoturísticas en torno al patrimonio cultural y religioso. Esta combinación podría reforzar tanto el turismo de recreación y naturaleza como el religioso al potenciar los atractivos religiosos y culturales merideños mediante la combinación de los mismos con atracciones de recreación capaces de alargar la estadía de una mayor cantidad de turistas y por lo tantogenerar una mayor cantidad de ingresos para el destino.

En un primer sentido es una excelente oportunidad para reforzar el turismo cultural y religioso, ofreciendo experiencias y vivencias a los visitantes; por ejemplo, la naturaleza como atractivo del turismo tradicional (recreacional, de aventura o montaña) podría formar parte de la experiencia espiritual, de tranquilidad, meditación y oración para el turista religioso a la vez que del turismo de descanso (Shackley, 2002; Ya-Ping Chang and Kuang-Peng, 2021).

Estas rutas podrían generar un tejido de múltiples emprendimientos no solo a nivel de programas sociales sino a nivel empresarial para la respectiva dotación de equipamiento de las rutas, capaces de apoyar y hacer funcional turísticamente el patrimonio histórico y cultural del destino en el marco del ecoturismo y del turismo sostenible; además de satisfacer a una considerable cantidad de turistas de nivel educativo alto, lo que podría incluir emprendimientos gastronómicos (dulcería, bebidas) y toda manifestación religiosa y autóctona del Estado. Se sugieren proyectos de reubicación de mercados informales, el desarrollo de sistemas de transporte, la disposición de desechos sólidos y la restauración de casonas, iglesias, plazas, parques, instituciones públicas y monumentos deteriorados o subutilizados, el desarrollo de programas educativos parainvolucrar a la comunidad receptora para conocer e interesarse por las potencialidades turísticas patrimoniales histórico-culturales de la ciudad y la formación de guías capaces de emitir información precisa y veraz sobre ese patrimonio. 


\section{Referencias}

Argyriadis, K. (2008). Speculators and santuristas: The development of Afro-Cuban cultural tourism and the accusation of religious commercialism. TouristStudies, 8(2), 249-265.

Aulet, S. y Hakobyan, A. (2011). Turismo religioso y espacios sagrados: una propuesta para los santuarios de Catalunya. RITUR. Revista Iberoamericana de Turismo, 1(1), 63-82.

Boada, I. (2018). Patrimonio religioso en la propuesta de rutas geoturísticas para la Ciudad de Mérida. En II Congreso Interdisciplinario del Estado Mérida: entre lo cultural y el desarraigo, Mérida, Venezuela.

Carrizo, A.y Vieira, A. (2009). Turismo de eventos: desafíos estratégicos de la ciudad de João Pessoa (Brasil). Cuadernos de Turismo, 23, 3146.

Collins, N. (2010). Geographers and Pilgrimages: changing concepts in pilgrimagestourism research. Tijdschrift voor Economische en Sociale Geografie, 101(4), 437-448.

Collins-Kreiner, N. and Kliot, N. (2000). Pilgrimage tourism in the holy land: The behavioral characteristics of Christian pilgrims. GeoJournal, 50(1), 55-67.

Collins-Kreiner, N. (2010). Current Jewish pilgrimage tourism: Modes and models of development. Tourism Preliminary Communication, 58(3), 259-270.

Crouch, G. (1994). The Study of International Tourism Demand: A Survey of Practice.Journal of Travel Research, 32(4), 41-57.

Garau-Vadell, J., Gutierrez-Taño, D. and Diaz-Armas, R. (2018). Economic crisis and residents' perception of the impacts of tourism in mass tourism destinations. Journal of Destination Marketing \& Management, 7, 68-75.

García, R.y Olmos, L. (2015). Estructura del mercado turístico. Madrid, España: Paraninfo.
Goeldner, C. y Ritchie, B. (2011). Turismo: planeación, administración y perspectivas. Ciudad de México, México: LIMUSA.

Guillén, A. y Pérez, R. (2016). Estudio geoturístico del patrimonio histórico-cultural de la ciudad de Mérida. Mérida, Venezuela: Universidad de Los Andes.

Hsin-Yu, M. (2021). Exploring the motivationbased typology of religious tourists: A study of Welcome Royal Lord Festival. Journal of Destination Marketing \& Management, 21, 100623.

Hughes, G. (1995). The cultural construction of sustainable tourism. Tourism Management, 16(1), 49-59.

Mariani, M. et al. (2014). Managing change in tourism destinations: key issues and current trends. Journal of Destination Marketing \& Management, 2(4), 269-272.

Maxim, C. and Chasovschi, C. (2021). Cultural landscape changes in the built environment at World Heritage Sites: Lessons from Bukovina, Romania. Journal of Destination Marketing \& Management, 20, 100583.

McIntosh, R.W. and Goeldner, C.R. (1986). Tourism: principles, practices, philosophies. New York, USA: John Wiley \& Sons.

Morére, N. y Perelló, S. (2013). Turismo cultural: patrimonio, museos y empleabilidad. Madrid, España: Fundación EOI.

Morillo, M. (2014). Sistema turístico del Estado Mérida, Venezuela: elementos de oferta. Revista Economía, XXXIX(38), 111-142.

Morillo, M. (2018). Turismo de eventos: alternativa de diversificación de la oferta turística del Estado Mérida, Venezuela. Actualidad Contable, 21(37), 118-150.

Mostafa-Rasoolimanesh, S. et al. (2011). Understanding memorable tourism experiences and behavioural intentions of heritage tourists. Journal of Destination Marketing \& Management, 21, 100621. 
Nieminem, K. (2012). Religious tourism: a finish perspective (tesis de posgrado). Vantaa, Finland: University of Applied Sciences.

OMT. (2018). Panorama OMT del turismo internacional. Recuperado de h t tp s: / / www.e-unwto.org / d o i / pdf/10.18111/9789284419890.

OMT. (2020). Barómetro OMT del turismo mundial y anexo estadístico. Recuperado de https:// www.e-unwto.org/doi/pdf/10.18111/ wtobarometeresp.2020.18.1.1.

Pérez, L. (2014). Turismo cultural: una oportunidad para el desarrollo local. Guía Metodológica. Santiago de Chile, Chile: Servicio Nacional de Turismo.

Sancho, A. (2006). Introducción al turismo. Organización Mundial del Turismo (OMT). Madrid, España: Ministerio de Comercio, Industria y Turismo.

Shackley, M. (2002). Space, Sanctity and Service, the English Cathedral as Heterotopia. International Journal of Tourism Research, 4(5), 345-352.

Shinde, K.A. (2010). Entrepreneurship and Indigenous Enterpreneurs in Religious Tourism in India. Internacional Journal of Tourism Research, 12(5), 523-535.

Swarbrooke, J. (1995). The Development and Management of Visitor Attractions. London, England: Butterworth Heinamann.

Tobón, S. y Tobón, N. (2013). Turismo religioso: fenómeno social y económico. Turismo y Sociedad, 14, 237-249.

Velasco, M. (2009). Gestión turística del patrimonio cultural: enfoques para un desarrollo sostenible del turismo cultural. Murcia, España: Universidad de Murcia.

Wang, D., Xiang, R. and Yunpeng, Li. (2013). China's "smart tourism destination" initiative: A taste of the service-dominant logic. Journal of Destination Marketing \& Management, 2(2), 59-61.
Ya-Ping Chang, A. and Kuang-Peng, H. (2021). Development and validation of a tourist experience scale for cultural and creative industries parks. Journal of Destination Marketing \& Management, 20, 100560. 